\title{
Finding common ground
}

\section{Two competing hypotheses about Parkinson's and Gaucher's diseases need to be knitted together, say Anthony H. Futerman and John Hardy.}
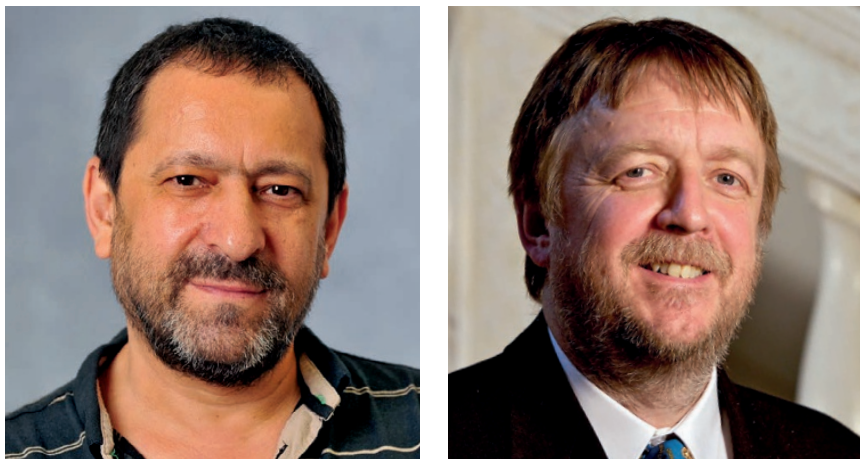

G aucher's disease is rare, despite being the most common of the lysosomal storage disorders (LSDs) ${ }^{1}$. This family of diseases is characterized by defects in any of the genes that encode proteins that the lysosome needs to perform its functions ${ }^{2}$, chief of which is the degradation of unwanted cellular material. In the case of Gaucher's disease, the GBA1 gene is faulty. The gene encodes the glucocerebrosidase protein, which normally degrades the lipid glucosylceramide. But in people with two defective copies ('alleles') of the gene, the mutant glucocerebrosidase cannot adequately degrade the glucosylceramide, which accumulates in lysosomes throughout the body.

The symptoms of Gaucher's disease vary according to which of the three variants the person has. Around $90 \%$ of people with Gaucher's have type 1 , in which symptoms include an enlarged liver and spleen; the remainder have either type 2 or 3 , in which neurological effects are also seen to different degrees, often leading to death at a young age. Gaucher's affects 1 person in around 40,000 to 60,000, although certain ethnic groups, such as Ashkenazi Jews, have a much higher incidence.

By contrast, Parkinson's disease is the second most common neurodegenerative disorder, after Alzheimer's disease, and affects about $1 \%$ of people over the age of 65. People with Parkinson's disease display symptoms ranging from muscle rigidity and tremors to changes in speech and gait. The cause of Parkinson's remains unknown, but patients' brains typically have abnormal accumulation of a protein known as $\alpha$-synuclein. Parkinson's disease is not caused by a fault in one specific gene, instead several genes are known to predispose an individual towards developing the disease up to one in five patients have a family history of Parkinson's ${ }^{3}$.

In many ways, these two diseases seem to be entirely distinct from one another. But there is a puzzling, and quite unexpected, link: work over the past decade or so has shown that mutations in one allele of $G B A 1$ are the most frequent genetic risk factor for Parkinson's disease ${ }^{4}$. However, there do not seem to be any common symptoms between Parkinson's and Gaucher's diseases: people with type 1 Gaucher's disease do not display any obvious signs of neurological impairment, and the neurological problems of those with types 2 and 3 are very different from those seen in Parkinson's. Similarly, individuals with a mutation in only one copy of GBA1 do not display symptoms (even mild ones) of Gaucher's disease and are considered healthy.

\section{GAIN OR LOSE}

The relationship between Gaucher's and Parkinson's diseases is mired in controversy. It is unclear how the effects of the mutated GBA1 gene might provide a mechanism that underlies the two diseases. There are two contrasting hypotheses: one that relies on the mutant glucocerebrosidase failing to adequately perform its normal role (loss of function), and another where, as well as this failure, the mutant enzyme also acquires a new activity (gain of function). Navigating through the evidence for and against each hypothesis has become exceedingly difficult.

The gain-of-function hypothesis is based on the idea that the mutated GBA1 gene encodes a version of glucocerebrosidase that does not fold correctly. This improperly structured protein misbehaves in two ways: as well as being unable to adequately clear glucosylceramide, it also interacts with new cellular pathways. Several brain diseases are associated with similar defects in protein folding, including Creutzfeldt-Jakob disease, Alzheimer's disease and Parkinson's.

Animal and cellular models of Gaucher's disease show that a substantial pool of mutated glucocerebrosidase protein does indeed misfold after being generated in the endoplasmic reticulum - the cellular organelle responsible for protein assembly. And studies have shown that mutated glucocerebrosidase is present in a significant proportion of a-synuclein aggregates in the brains of animal models of both Parkinson's and Gaucher's diseases ${ }^{5}$. These findings suggest that misfolded glucocerebrosidase could contribute to the development of Parkinson's disease, either by increasing the aggregation of $a$-synuclein or by interfering with its clearance. Mutated proteins are normally eliminated through the endoplasmic reticulum; mutant glucocerebrosidase might accumulate here and overwhelm the clearance pathway, allowing $\alpha$-synuclein to build up.

The competing loss-of-function hypothesis suggests that the deleterious consequences of both Gaucher's and Parkinson's diseases can be sufficiently explained by the lack of enzyme activity and the consequent accumulation of glucosylceramide lipid in the lysosome.

Evidence supporting this hypothesis comes from people who have specific mutations in one copy of GBA1 that prevent any glucocerebrosidase from being expressed; these people can still develop Parkinson's disease. Equally, mice that have been engineered to not express any glucocerebrosidase ${ }^{6}$ will still show symptoms associated with Gaucher's disease, and in some cases also show defects in areas of the brain that are commonly associated with Parkinson's disease. Studies in cell and in animal models in which glucocerebrosidase protein is 
chemically inhibited, which does not cause any protein misfolding, can still result in the accumulation of $\alpha$-synuclein. Together, these data suggest that both diseases can develop even in the absence of misfolded protein.

\section{BALANCING ACT}

Other lines of evidence must also be considered when weighing the two hypotheses against each other. For instance, the link between Parkinson's and Gaucher's diseases might well extend to other LSDs ${ }^{7}$. Mutations in several other genes that encode various lysosomal proteins also seem to predispose individuals to Parkinson's disease. One of the clearest and most recent examples uncovered is the lysosomal protein sphingomyelinase, which is encoded by the SMPD1 gene that is responsible for Niemann-Pick disease ${ }^{8}$. However, mutations in other lysosomal proteins have been studied much less than glucocerebrosidase because the LSDs they cause are much rarer than Gaucher's disease.

If several genes that compromise lysosome function can lead to Parkinson's disease, this suggests that the link between GBA1 and Parkinson's might not be specific to a gain of function for glucocerebrosidase, but rather to the lysosome's role more broadly. A leading candidate is autophagy - the process by which the lysosome recycles cellular components, such as defunct mitochondria. Dysfunction in autophagy is associated with many $\mathrm{LSDs}^{9}$ and several late-onset neurodegenerative diseases, including Parkinson's disease ${ }^{10}$. Whatever the pathogenic pathway, it may well be that once one aspect of lysosomal function is compromised, there is a short path to neuronal dysfunction and, eventually, a neurodegenerative disease.

Central to these hypotheses is the idea that the lysosome seems to be able to function adequately if there is a mutation in just one allele of GBA1. There is little evidence, for instance, of any accumulation of glucosylceramide in people with Parkinson's disease who have a mutation in only one GBA1 allele; such individuals do not show any overt symptoms reminiscent of those in Gaucher's disease. But the key word here might be 'overt'. Neurodegenerative diseases typically occur in older individuals, so perhaps even subtle, barely measurable changes in lysosome biology will predispose an individual to a neurodegenerative disease. This concept is supported by observations that several cellular organelles, including the lysosome, become less effective with age ${ }^{11}$. It might take only small changes in the accumulation of substances in the lysosome to induce neurodegenerative diseases later in life.

\section{JOINING UP}

The stage is set for an investigation of these two competing hypotheses. In one scenario, misfolded glucocerebrosidase causes $\alpha$-synuclein to aggregate in a protein-led disease. In the other scenario, dysfunctional glucocerebrosidase induces a change in lysosomal function that over time results in Parkinson's disease. Both hypotheses can partly explain the molecular association between Parkinson's and Gaucher's diseases, but neither can adequately explain why mutations in only one copy of the GBA1 gene predispose a person towards Parkinson's disease. The truth is likely to involve some combination of these two seemingly contradictory ideas.

Better use of animal models of the two diseases could help to resolve the situation. Mice with a mutation in only one GBA1 allele that seem to be healthy have not been the subject of intense investigation up to now, but they could reveal important clues. Mice that have mutations in both alleles, and so have Gaucher's disease, rarely live long enough to develop Parkinson's. Use of a chemical inhibitor of glucocerebrosidase could help researchers to develop longer-living models of Gaucher's disease. A recent study ${ }^{12}$ that used this approach has led to the discovery of a number of genes that might modify the severity of both Gaucher's and Parkinson's diseases.

In humans, more post-mortem data could help in the hunt for

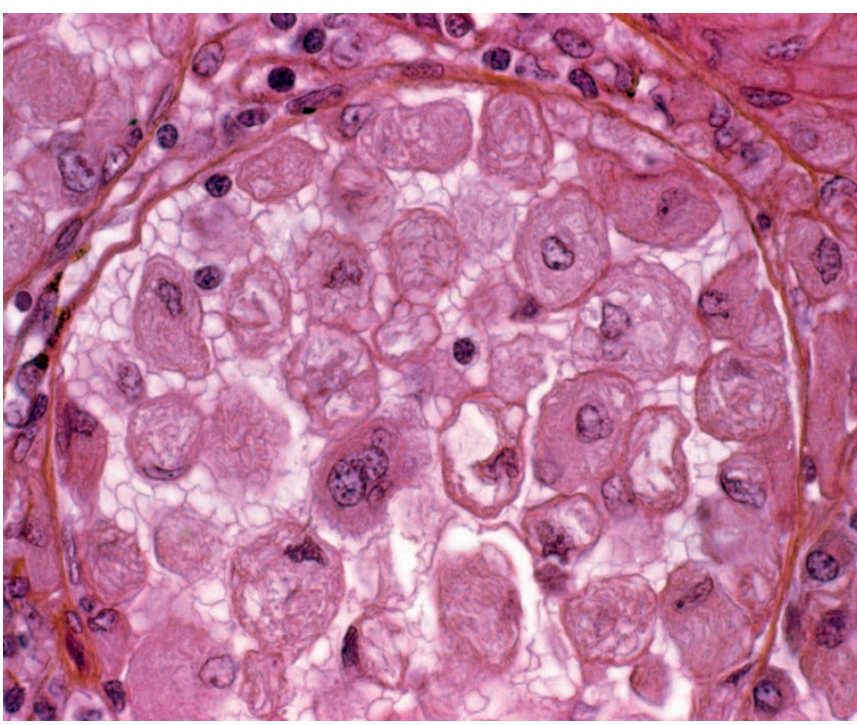

Spleen cells in Gaucher's disease contain so much glucocerebroside that they do not stain as dark as normal cells (top right).

similarities between the two diseases. The brains of people with type 1 Gaucher's disease, who tend to die old, are rarely studied. But given the presence of $\alpha$-synuclein aggregates, more work could reveal whether there are any links with Parkinson's disease.

Genetic studies are likely to provide several of the missing pieces, including confirmation of whether there is indeed an association between mutations in other lysosomal proteins and Parkinson's disease. Such studies are not easy to do. Not only do they require large numbers of patients' DNA samples - which are very difficult to acquire for rare diseases such as LSDs - but researchers also need to be able to sequence large regions of the human genome.

For GBA1 specifically, a rewarding line of study might be to focus on individuals who have only one mutant allele. At the moment, these people are considered healthy (at least, those without Parkinson's disease) and hence not worthy of special study. But they should not be difficult to find: the Ashkenazi Jewish population, for instance, has a high rate of carriers.

It has been about a decade since the GBA1 link between Gaucher's and Parkinson's diseases was first unearthed. Researchers on both sides have come together to try to understand the underlying mechanism, but have essentially got no further than these two hypotheses. A concerted effort is needed by geneticists, cell biologists, neurobiologists and clinicians to gather samples from patients and carriers to finally resolve the fascinating, yet currently perplexing, relationship between neurodegenerative diseases and the lysosome.

Anthony H. Futerman is at the Department of Biomolecular Sciences, Weizmann Institute of Science, Rehovot, Israel, and John Hardy is at the Institute of Neurology, University College London. e-mail:Tony.Futerman@weizmann.ac.il and j.hardy@ucl.ac.uk

1. Futerman, A. H. \& Zimran, A. Gaucher Disease (CRC Press, 2006)

2. Futerman, A. H. \& van Meer, G. Nature Rev. Mol. Cell Biol. 5, 554-565 (2004).

3. Hardy, J. Neuron 68, 201-206 (2010)

4. Sidransky, E. et al. N. Engl. J. Med. 361, 1651-1661 (2009).

5. Sardi, S. P., Singh, P., Cheng, S. H., Shihabuddin, L. S. \& Schlossmacher, M. G. Neurodegen. Dis. 10, 195-202 (2012)

6. Farfel-Becker, T. et al. Hum. Mol. Genet. 23, 843-854 (2014).

7. Shachar, T. et al. Move. Disord. 26, 1593-1604 (2011).

8. Dagan, E. et al. Parkinson. Relat. Disord. 21, 1296-1297 (2015).

9. Lieberman, A. P. et al. Autophagy 8, 719-730 (2012).

10.Nixon, R. A. Nature Med. 19, 983-997 (2013).

11.Rocha, E. M. et al. Ann. Clin. Transl. Neurol. 2, 433-438 (2015).

12.Klein, A. D. et al. Cell Rep. http://dx.doi.org/10.1016/j.celrep.2016.07.085 (2016). 